\title{
Clinical effect of Non-Thermal CO2 Laser Therapy (NTCLT) on Pain Relief of Oral Aphthous Ulcers of Behçet's Disease
}

\author{
Nasrin Zand ${ }^{*(\oplus}$, Leila Ataie-Fashtami ${ }^{2}$, Parvin Mansouri ${ }^{3}{ }^{\circledR}$ Mohsen Fateh $^{4}{ }^{\circledR}$, Afshan Shirkavand $^{1}{ }^{\circledR}$ \\ ${ }^{1}$ Department of Medical Laser, Medical Laser Research Center, Yara institute, Academic Center for Education, \\ Culture and Research (ACECR), Tehran, Iran \\ ${ }^{2}$ Department of Regenerative Medicine, Royan Institute for Stem Cell Biology \& Technology, Academic Center for \\ Education, Culture and Research (ACECR), Tehran, Iran \\ ${ }^{3}$ Research Vice-President of Medical Laser Research Center, Yara institute, Academic Center for Education, Culture \\ and Research (ACECR), Tehran University of Medical Sciences (TUMS), Tehran, Iran \\ ${ }^{4}$ Research Fellow, Life Style \& Health Management Department, Academic Center for Education, Culture and \\ Research (ACECR), Tehran, Iran
}

\section{*Correspondence to Nasrin Zand, MD; Assistant Professor of Dermatology, Department of Medical Laser, Medical Laser Research Center, Yara institute. ACECR. No.17, Nazari Street, Abourihan Street, Enqelab Avenue, Tehran Iran. Tel: +982166462915 +989129359096, Email: zand nas@yahoo.com}

Received: March 6, 2021 Accepted: August 14, 2021 Published online November 7 , 2021

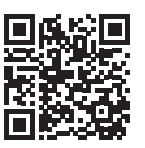

\begin{abstract}
Introduction: Behçet's disease (BD) is a debilitating and chronic vasculitis which can affect multiple organs. Recurrent oral aphthosis is the most common clinical feature of BD. The remarkable pain associated with painful oral ulcers can exert a major impact on the patients' quality of life. Relieving pain of these oral ulcers is an essential approach in the management of mucocutaneous lesions of BD. Non-thermal CO2 laser therapy (NTCLT) as a novel photobiomodulative approach has recently been used for significant and immediate pain reduction of some types of painful oral lesions such as recurrent aphthous stomatitis, pemphigus vulgaris, and so on. In this procedure by some considerations, the conventional CO2 laser is used as a non-surgical, non- thermal, photobiomodulative laser without any visible thermal adverse effects. In this article, we report our experiences about the analgesic effects of NTCLT on painful oral aphthous ulcers of BD.

Case Presentation: We report four cases of BD, whose painful oral aphthous ulcers were irradiated with NTCLT. Immediately after NTCLT, the pain of the lesions was significantly relieved with no visible complications.

Conclusion: The results of this study suggest that NTCLT could be proposed as a hopeful procedure for significant and instant pain relief of oral aphthous ulcers of BD without any visible thermal adverse effects.

Keywords: Behcet's disease; Non-thermal CO2 laser therapy (NTCLT); Oral aphthous ulcers; Pain relief; Photobiomodulation.
\end{abstract}

\section{Introduction}

Behçet's disease (BD) is a chronic, relapsing, multisystem vasculitis which can affect vessels of all sizes of various organs. Oral ulcers, genital ulcers, skin lesions, and articular and ocular involvement are the most common manifestations of $\mathrm{BD} .^{1-8} \mathrm{BD}$ has global distribution; however, it is particularly prevalent along the "ancient Silk Road”, with the highest prevalence in Turkey, Iran, Japan, China and Korea. In Iran, the prevalence of BD has been estimated to be $16-80$ patients per 100000 residents. ${ }^{2}$

Recurrent oral aphthosis which often precedes other clinical signs is the most common mucocutaneous manifestation of BD observed in more than $90 \%$ of cases. $^{8-11}$ The frequency of oral aphthosis in a large series of patients within the Iranian nationwide registry was $97.3 \% .^{3}$ These lesions with a diameter of 1-20 mm are painful, punched-out ulcers with grayish yellow necrotic base, surrounded with a bright red halo. ${ }^{1-8}$ The ulcers usually subside within 1 to 4 weeks spontaneously and recur at intervals from days to months. ${ }^{2,4}$ The considerable pain of these ulcers may cause difficulty during eating, drinking and performing routine oral hygiene and may exert a major impact on the patients' quality of life. ${ }^{9-11}$ Since the definite mechanisms of oral ulcers in $\mathrm{BD}$ are poorly understood, the treatment of oral ulcers (without any other organ involvement) is mainly aimed at pain relief, the promotion of the healing process and the reduction of the recurrence rate. ${ }^{9-11}$ These lesions

Please cite this article as follows: Zand N, Ataie-Fashtami L, Mansouri P, Fateh M, Shirkavand A. Clinical effect of non-thermal co2 laser therapy (NTCLT) on pain relief of oral aphthous ulcers of behçet's disease. J Lasers Med Sci. 2021;12:e72. doi:10.34172/jlms.2021.72. 
are usually painful, hindering the patients from eating, drinking and speaking. Due to the relationship between pain and quality of life, obtaining new pain-relieving approaches for the symptomatic management of these painful ulcers during conventional treatment is rational.

Over the past decades photobiomodulation (PBM) has gained increasing acceptance as a beneficial, nonpharmacological therapeutic modality to relieve pain, reduce inflammation and stimulate the healing process. The most common wavelengths employed in PBM are in the red and near-infrared spectral regions. However, recent studies have indicated that by some measurements, the CO2 laser $(10600 \mathrm{~nm})$ can be used as a low-level, nonthermal, photobiomodulative laser to achieve remarkable and immediate pain relief on some oral and mucosal lesions with no visible adverse effects. This process is non-thermal, smokeless, and pain-free with no need for anesthetic agents. ${ }^{12-20}$ Afterwards this procedure was termed NTCLT (non-thermal CO2 laser therapy). In our previous studies, we reported the significant and instant pain-relieving effects of NTCLT on some types of painful oral and mucosal lesions such as recurrent aphthous stomatitis, oral lesions of pemphigus vulgaris and genital ulcers of BD. ${ }^{14-16,19-20}$ In this paper, we report 4 cases of $\mathrm{BD}$, whose painful oral ulcers immediately responded to the pain-relieving effects of NTCLT without any visible thermal complications.

\section{Clinical Cases \\ Case 1}

A 36-year-old female patient with $\mathrm{BD}$ presented to the laser clinic of Medical Laser Research Center, Yara Institute, for relieving the pain of her oral aphthous ulcer by NTCLT. She fulfilled the International Criteria for Behcet's disease (ICBD), with a 10-year history of recurrent oral aphthosis with high frequency, histopathologically proven genital aphthous ulcers and a positive pathergy test. She was treated with sulfasalazine and colchicine. On clinical examination, there was an aphthous ulcer in her upper lip (Figure 1), posing difficulty in her eating and speech. She was requested to grade and record the contact VAS (visual analog scale) pain scores of her ulcer on a horizontal 10$\mathrm{cm}$ line before and immediately after NTCLT, 4 and 8 hours after the operation and then daily for the next 5 days.

Before NTCLT, the ulcer and its surrounding tissue were covered with a thick layer (3-4 mm) of transparent gel (Abzar Darman Co., Iran) with high water content (87.5\%) with no anesthetic effects. The surgical staff and the patient used protective eyeglasses during the procedure. The $\mathrm{CO}_{2}$ laser $(\lambda=10600 \mathrm{~nm}$; Lancet-2, Russia) was operated (power: $1 \mathrm{~W}$ ) with a de-focused handpiece, scanning the surface of the lesion with rapid circular motion of the handpiece. Immediately after NTCLT, the contact VAS pain score of the ulcer showed a

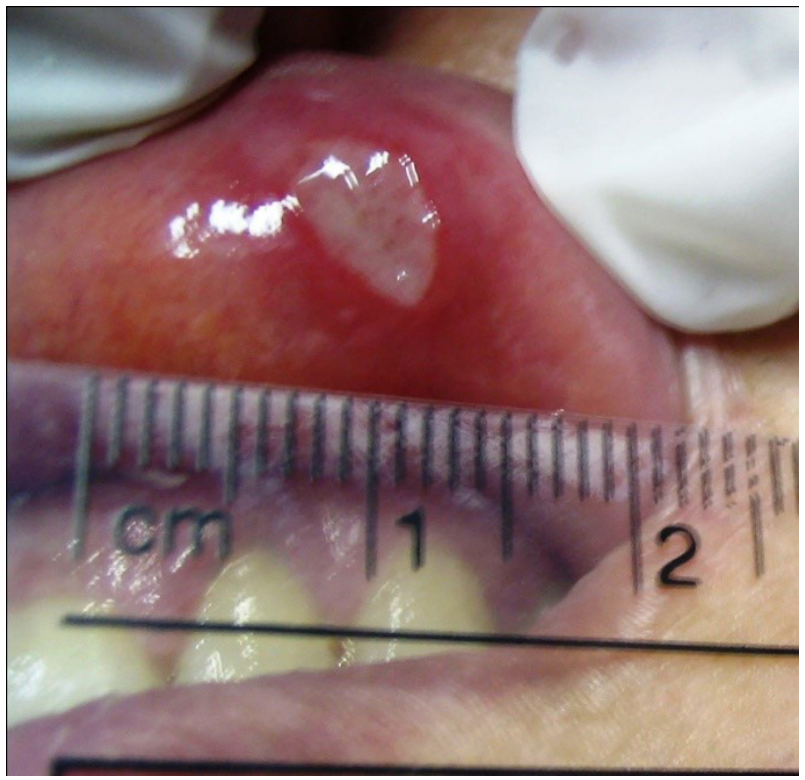

Figure 1. Case 1: Aphthous Ulcer of the Upper Lip Before NTCLT.

significant improvement from 6 to 0 . No kind of thermal side effects or aggravation of the lesion was observed after NTCLT. The procedure was pain-free and no kind of anesthetic agent was needed. The pain-relieving effect of NTCLT continued during the four-day healing period. The patient was visited every other day for four days and then two and four weeks after NTCLT. No kinds of visible complications such as burn or aggravation of the ulcers were detected during the patient's follow-up periods.

\section{Case 2}

A 34-year-old male patient with known BD was referred to our clinic for a palliative care consult and NTCLT of his painful oral ulcer. He fulfilled the ICBD, with a 12year history of recurrent oral aphthous ulcers with high frequency, genital aphthous ulcers and a positive pathergy test.

On clinical examination, there was an aphthous ulcer in his lower lip (Figure 2). The contact and non-contact VAS pain scores of the ulcer were 7 and 4 respectively.

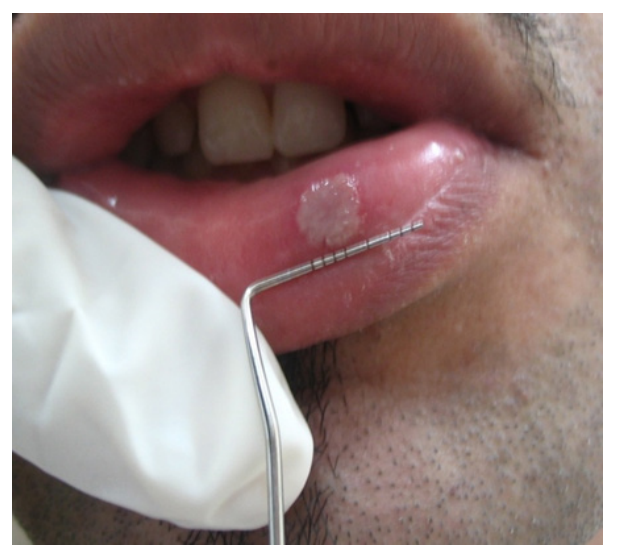

Figure 2. Case 2: Aphthous Ulcer of the Lower Lip Before NTCLT. 
The patient's aphthous ulcer was treated with NTCLT (with the same protocol as the first case). Immediately after NTCLT, the pain of the ulcer was relieved completely; therefore, he was able to drink and eat easily. No visible adverse effects were observed after NTCLT. The technique was pain-free with no need for anesthetic agents. The pain-relieving effect of NTCLT continued throughout the four days of the recovery period.

\section{Case 3}

A 34-year-old male patient with known BD was referred to our clinic for relieving the pain of his oral aphthous ulcer by NTCLT. He fulfilled the ICBD criteria for diagnosis of $\mathrm{BD}$, with a 12 -year history of recurrent oral aphthosis with high frequency, a history of erythema nodosum, ocular lesions, clinically consistent with BD, and a positive pathergy test.

The contact and non-contact VAS pain scores of his ulcer in the upper lip (Figure 3) were 7 and 3 respectively. Immediately after NTCLT both the contact and noncontact pains of the ulcer were completely relieved. However, after an hour the contact pain recurred (VAS contact pain score: 3 ), although it was less than the pretreatment level. The VAS contact pain score of the lesion remained 4 at 4 and 12 hours post-operatively. The patient recorded the contact VAS pain scores 3,2, 2 and 0 on the $2^{\text {nd }}, 3^{\text {rd }}, 4^{\text {th }}$ and $5^{\text {th }}$ days post-laser respectively. After NTCLT the non-contact pain score of the lesion was zero during the five-day healing period. No kind of complications was reported within the follow-up periods.

\section{Case 4}

A 47-year-old male patient with known BD was referred to our clinic for a palliative care consult and NTCLT of his painful oral ulcer. He met the ICBD criteria for diagnosis of $\mathrm{BD}$, with a history of recurrent oral and genital aphthous ulcers and a positive pathergy test. On clinical examination, there was an aphthous ulcer in his lower lip (Figure 4). The contact and non-contact VAS pain scores of his ulcers were 6 and 2 respectively. Immediately after NTCLT the pain of the ulcer was relieved completely. The

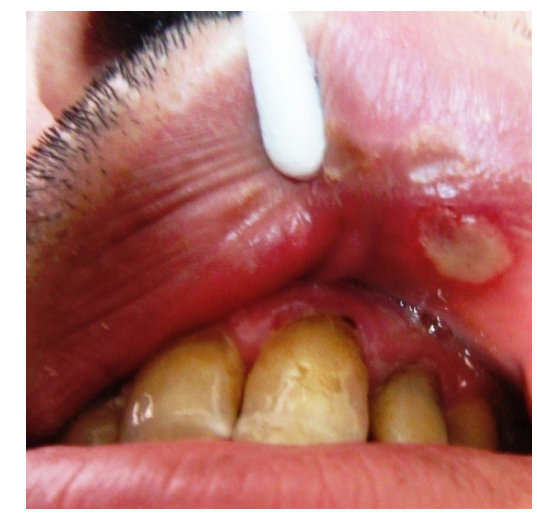

Figure 3. Case 3: Aphthous Ulcer of the Upper Lip Before NTCLT. lesion pain did not recur during the four-day healing time.

\section{Discussion}

The results of NTCLT in these patients suggest that NTCLT as a photobiomodulative procedure could be potentially considered a hopeful procedure for the immediate pain management of oral ulcers of BD without any visible thermal adverse effects. The application of NTCLT was painless and smokeless and no kind of anesthesia was required.

Over the past decades PBM (low-level laser therapy) has gained increasing acceptance as an efficient, nonpharmacological and safe therapeutic procedure for pain relief, inflammation reduction and improving and accelerating the wound healing process. A growing number of reports have demonstrated promising outcomes of PBM for the pain management of diverse acute and chronic painful conditions.

The most popular wavelengths applied for photobiomodulation therapy (PBMT) are in the red and near-infrared spectrum. However, some studies have pointed out that the far infrared wavelength (CO2 laser, $10600 \mathrm{~nm}$ ) can be applied as a non-ablative, non-thermal laser for PBM, ${ }^{12-20}$ a technique which was termed NTCLT afterwards. ${ }^{19,20}$

In addition to traditional photobiomodulative lowpower lasers, high-power surgical lasers can also be used for PBMT. As Tuner stated, "When high power lasers are used for biomodulation, one only need to make the beam wide enough not to burn. An alternative is to scan rapidly over the lesion with a narrow beam. Therefore, the power density or average power is kept low enough to avoid burning, and their incident energy and power density are set within the low intensity laser therapy range." 21

For the application of the CO2 laser for NTCLT as a photobiomodulative (low-level laser therapy) procedure, in addition to the rapid scan of the lesion with a defocused beam, illumination of the lesion is performed through a thick layer of a gel with high water content. By these measurements the final beam power significantly drops (200-500 times) on the surface of the gel to the level of

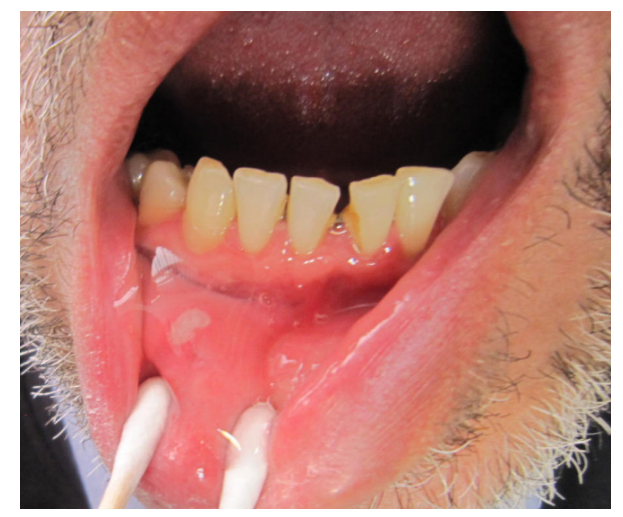

Figure 4. Case 4: Aphthous Ulcer of the Lower Lip Before NTCLT. 
low-intensity photobiomodulative lasers ${ }^{14}$ and can be successfully applied for the pain management of some oral lesions without any visible thermal adverse effects. This laser technique which was initially termed as non-ablative laser therapy, after proving its non-thermal nature, was renamed as NTCLT to avoid misunderstanding with surgical fractional nonablative $\mathrm{CO} 2$ lasers used by dermatologists for cosmetic purposes. ${ }^{19,20}$

In this article, we reported known cases of $\mathrm{BD}$, whose painful oral aphthous ulcers were illuminated with NTCLT and immediately after the procedure, the pain of the lesions was relieved dramatically without any visible side effects even erythema. As we guessed before, none of the patients reported warmth sensation in their ulcers throughout NTCLT, and the procedure was pain-free and did not require anesthesia.

During the performance of NTCLT procedure, we highly recommend the application of eyeglasses not only matched to the CO2 laser (10 $600 \mathrm{~nm}$ ) wavelength but also to the wavelength of the guiding beam to protect the physician's eyes against the reflected beam from the surface of the gel.

Since BD is a serious systemic disease with considerable morbidity and even mortality, the patients should be informed that they should stay on their prescribed systemic drugs for $\mathrm{BD}$ in spite of the significant and instant analgesic effects of NTCLT. This report can be valuable for dentists, dermatologists and rheumatologists. There is a clear need for well-designed high quality controlled studies with large sample sizes to prove the pain-relieving effects of NTCLT on oral aphthous ulcers of $\mathrm{BD}$. In addition, these kinds of clinical trials will be able to demonstrate whether NTCLT can improve and accelerate the wound healing process of these lesions or not.

\section{Conclusion}

The results of this report suggest that NTCLT could be potentially proposed as a hopeful procedure for instant and considerable pain relief of oral aphthous ulcers of BD without any visible complications.

\section{Ethical Considerations}

Informed consent for the execution of the procedure and for the scientific use of images was obtained from the patients.

\section{Conflict of Interests}

The authors declare no conflict of interest.

\section{References}

1. Alpsoy E. Behcet's disease: A comprehensive review with a focus on epidemiology, etiology and clinical features, and management of mucocutaneous lesions. J Dermatol. 2016; 43(6): 620-32. doi: 10.1111/1346-8138.13381.

2. Davatchi F, Shahram F, Chams-Davatchi C, Shams H, Abdolahi BS, Nadji A, Faezi T, Akhlaghi M, Ghodsi Z, Karimi N, Kavosi H, Mohtasham N, Masoumi M,
Shadmanfar S, Mousavi M. Behcet's disease in Iran: Analysis of 7641 cases. Mod Rheumatol. 2019; 29(6):10231030. doi: 10.1080/14397595.2018.1558752.

3. Davatchi F. Behçet's disease. Int J Rheum Dis. 2018; 21(12):2057-2058. doi: 10.1111/1756-185X.13465.

4. Bettiol A, Prisco D, Emmi G. Behçet: the syndrome. Rheumatology (Oxford). 2020; 59(Supple 3):iii101-iiil07. doi: 10.1093/rheumatology/kez626

5. Davatchi F, Chams-Davatchi C, Shams H, Shahram F, Nadji A, Akhlaghi M. Behcet's disease: epidemiology. Clinical manifestations and diagnosis. Expert ev Clin Immunol. 2017; 13(1): 57-65. doi: 10.1080/1744666XX.2016.1205486

6. Giannetti L, Murri Dello Diago A, Lo Muzio L. Behçet's disease: minireview with emphasis on oral aspects. Minerva Stomatol. 2018;67(6): 246-249.doi: 10.23736/ S0026-4970.18.04135-3

7. Zeidan MJ, Saadoun D, Garrido M, Klatzmann D, Six A, Cacoub P. Behçet's disease physiopathology: a contemporary review. Auto Immun Highlights. 2016; 7(1): 4. doi: 10.1007/s13317-016-0074-1.

8. Alpsoy E, Zouboulis CC, Ehrlich GE. Mucocutaneous lesions of Behcet's disease. Yonsei Med J. 2007; 48(4): 57385. doi: 10.3349/ymj.2007.48.4.573

9. Leccese P, Ozguler Y, Christensen R, Esatoglu SN, Bang D, Bodaghi B, et al. Celik AF, Management of skin, mucosa and joint involvement of Behçet's syndrome: A systematic review for update of the EULAR recommendations for the management of Behçet's syndrome. Semin Arthritis Rheum. 2019; 48(4):752-762. doi: 10.1016/j.semarthrit.2018.05.008

10. Karadag O, Bolek EC. Management of Behcet's syndrome. Rheumatology (Oxford). 2020; 59(Suppl 3): iii108-iiil17. doi: 10.1093/rheumatology/keaa086.

11. Taylor J, Glenny AM, Walsh T, Brocklehurst P, Riley P, Gorodkin R. Cochrane Database Syst Rev. 2014;2014(9):CD011018. Published 2014 Sep 25. doi:10.1002/14651858.CD011018.pub2

12. Elad S, Or R, Shapira MY, Haviv A, Galili D, Garfunkel AA, et al. CO2 laser in oral graft-versus-host disease: a pilot study. Bone Marrow Transplant. 2003; 32(10):1031-4. doi: 10.1038/sj.bmt. 1704272

13. Sharon-Buller A, Sela M. CO2-laser treatment of ulcerative lesions. Oral Surg Oral Med Oral Pathol Oral Radiol Endod. 2004; 97(3): 332-4. doi: 10.1016/j.tripleo.2003.11.012

14. Zand N, Ataie-Fashtami L, Djavid GE, Fateh M, Alinaghizadeh MR, Fatemi SM, Arbabi-Kalati F. Relieving pain in minor aphthous stomatitis by a single session of non-thermal carbon dioxide laser irradiation. Lasers Med Sci.2009; 24(4): 515-20. doi: 10.1007/s10103-008-0555-1.

15. Zand N, Fateh M, Ataie-Fashtami L, Djavid GE, Fatemi SM, Shirkavand A. Analgesic effects of single session of NonAblative CO2 Laser Therapy (NACLT) on major aphthous ulcers: (a preliminary study) Lasers Med. 2009; 6(4): 6-12.

16. Zand N, Fateh M, Ataie-Fashtami L, Djavid GE, Fatemi SM, Shirkavand A. Promoting wound healing in minor recurrent aphthous stomatitis by non-thermal, nonablative CO (2) laser therapy: a pilot study. Photomed Laser Surg, 2012; 30(12): 719-23. doi: 10.1089/pho.2012.3301. Epub 2012 Oct 31

17. Prasad RS, Pai A. Assessment of immediate pain relief with laser treatment in recurrent aphthous stomatitis. Oral Surg Oral Med Oral Pathol Oral Radiol. 2013; 116(2): 189-93. doi: 10.1016/j.oooo.2013.02.011. Epub 2013 Apr 23

18. Rezvaninezhad R S, Navabi N Z, Atai A, Shahravan Z. The 
effect $\mathrm{CO} 2$ laser on reducing pain associated with aphthous stomatitis. J Babol Univ Med Sci. 2016.18(10): 20-25

19. Zand N, Mansouri P, Fateh M, Ataie-Fashtami L, Rezaee Khiabanloo S, Safar F, et al. Relieving Pain in Oral Lesions of Pemphigus Vulgaris Using the Non-ablative, Nonthermal, CO2 Laser Therapy (NTCLT): Preliminary Results of a Novel Approach. J Lasers Med Sci. 2017; 8(1): 7-12. doi: 10.15171/jlms.2017.02
20. Zand N, Mansouri P, Rezaee Khiabanloo S, Fateh M, AtaieFashtami L, Safaee Naraghi Z, et al. The Immediate PainRelieving Effects of Non-Thermal CO2 Laser Therapy on Genital Ulcers of Behcet's Disease: A Case Report. J Lasers Med Sci. 2020;11(1):108-111. doi: 10.15171/jlms.2020.18

21. Tuner J, Hode L. Biostimulation, Laser therapy with high output lasers. In: The New Laser Therapy Handbook. Sweden: Prima books; 2010. 